\title{
Evaluation of Computed Tomography Findings in Revision Endoscopic Sinus Surgery
}

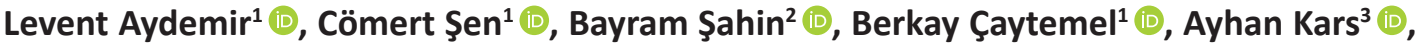 \\ Şenol Çomoğlu ${ }^{1}$, Meryem Nesil Keleş Türel ${ }^{1}$ (D)
}

\author{
${ }^{1}$ Istanbul University, Istanbul Faculty of Medicine, Department of Otolaryngology \& Head and Neck Surgery \\ ${ }^{2}$ Kocaeli Health Sciences University, Derince Training and Research Hospital, Department of Otorhinolaryngology-Head and Neck Surgery, Kocaeli, Turkey \\ ${ }^{3}$ Kastamonu University School of Medicine, Department of Otorhinolaryngology Kastamonu, Turkey
}

ORCID ID: L.A. 0000-0002-5836-4304; C.Ş. 0000-0002-5101-8599; B.Ş. 0000-0002-3886-4432; B.Ç. 0000-0002-8608-8749; A.K. 0000-0003-4580-315X; S..Ç. 0000-0003-4632-9218; M.N.K.T. 0000-0003-1829-8186

Citation: Aydemir L, Sen C, Sahin B, Caytemel B, Kars A, Comoglu S, Keles Turel MN. Evaluation of computed tomography findings in revision endoscopic sinus surgery. Tr-ENT 2021;31(3):55-59. https://doi.org/10.26650/Tr-ENT.2021.957490

\section{ABSTRACT}

Objective: To investigate computerized tomography (CT) data of patients who needed revision surgery for chronic rhinosinusitis.

Material and Methods: 83 patients who underwent revision endoscopic sinus surgery by the senior author due to recurrent and/or persistent chronic rhinosinusitis were included in this study. The following data were obtained from preoperative CT scans and recorded for each side of every patient: recurrent polyposis, incomplete uncinectomy, retained agger nasi cell, lateralization of middle turbinate, recirculation phenomenon, maxillary antrostomy stenosis, incomplete anterior and posterior ethmoidectomy, scarring at the frontal recess, sphenoid ostium stenosis, or novel onset sphenoid disease.

Results: Based on our findings, septal deviation caused inadequate posterior ethmoidectomy, while incomplete uncinectomy increased the risk of frontal sinus disease.

Conclusions: We suggest that septum deviation may cause insufficient visualization, while incomplete uncinectomy may prevent adequate intervention to the frontal sinus and these subsequently play a role in ESS failure.

Keywords: Revision FESS, sinus C.T scan, Functional endoscopic sinus surgery, rhinosinusitis

\section{INTRODUCTION}

Endoscopic sinus surgery (ESS) has been widely accepted for effective treatment of chronic rhinosinusitis and other inflammatory sinus diseases for which medical management has failed. Since its initial description by Kennedy in 1985 (1), reports on surgical success have ranged from $76 \%$ (2) to $97.5 \%$ (3). Despite the high surgical success rates, $10-15 \%$ of patients need revision surgery during long-term follow-up (4). Investigation of the factors that cause recurrent or persistent symptoms after primary surgery is required for successful management of revision cases. Several authors have tried to detect anatomic and systemic factors which may play a role in primary surgery failure. It has been reported that anatomical factors that may cause failure in primary surgery include recurrent polyps, lateralization of middle turbinate, incomplete uncinectomy, inappropriate maxillary antrostomy, scarring at the frontal recess, and scarring at the middle meatal antrostomy. Systemic factors include allergic rhinitis, cystic fibrosis, Samter's Triad, ciliary abnormalities, Kartagener's syndrome and other systemic inflammatory diseases $(2,3,5)$.

The main goal of the current study is to investigate computed tomography findings of patients who need revision surgery for chronic rhinosinusitis. This article does not focus on postoperative outcomes after revision sinus surgery.

\section{MATERIALS AND METHODS}

A retrospective review of patients undergoing endoscopic sinus surgery by the senior author from January 2012 to February 2017 identified 90 patients who underwent revision endoscopic

Corresponding Author: Berkay Çaytemel E-mail: drberkaycaytemel@gmail.com

Submitted: 30.06.2021 • Accepted: 01.09.2021 • Published Online: 23.09.2021

This work is licensed under Creative Commons Attribution-NonCommercial 4.0 International License. 
sinus surgery due to recurrent and/or persistent chronic rhinosinusitis. Patients with immune deficiency/suppression, cystic fibrosis, ciliary dysfunction, or history of previous Caldwell-Luc procedure and inferior meatal antrostomy were excluded from the study. Patients with incomplete information were also excluded and the remaining 83 patients were enrolled in the study.

All patients had been examined with nasal endoscopy and paranasal sinus CT scans (3-mm coronal, axial, and sagittal sections) preoperatively. Medical treatments included nasal corticosteroids, saline irrigations, decongestants, antibiotics, as well as management of allergy. Patients who have diffuse polyposis were also treated with systemic corticosteroids (1 $\mathrm{mg} / \mathrm{kg}$ daily). All surgeries were performed under general anesthesia and the surgical procedure performed was that defined by Kennedy $(1)$ and Stammberger $(6,7)$. During the surgical procedure the retained agger nasi cell was opened when required, residual uncinate process was removed, an incomplete anterior and/or posterior ethmoidectomy was completed, and frontal, maxillary and sphenoid ostium drainage was provided again. Absorbable packing with antibiotic ointment was put in the middle meatus at the end of surgery. Postoperatively, oral antibiotics and saline irrigations were administered for two weeks. Follow-up examinations were performed weekly during the first month after the surgery and absorbable packing remnants and crusts were removed with nasal endoscopy at the first visit.

The following data were evaluated and recorded for each side of every patient from preoperative CT scans: recurrent polyposis, incomplete uncinectomy, retained agger nasi cell, lateralization of middle turbinate, recirculation phenomenon, maxillary antrostomy stenosis, incomplete anterior and posterior ethmoidectomy, scarring at the frontal recess, sphenoid ostium stenosis, or novel onset sphenoid disease.

Ethics Committee Approval for the study was received from the Istanbul University School of Medicine Ethics Committee for Scientific Research (number 2021/415).

\section{RESULTS}

Among the 83 patients evaluated, 51 (61.4\%) were male and 32 (38.6\%) were female. The patients' ages ranged from 16 to 73 years, with the mean age being $45.4(+/-14.2)$ years. The mean number of previous surgeries was $1,9(+/-1,6)$ ( $\mathrm{min}$ : 1 - max: 9). Comorbid situations of patients are shown in Table 1. All sinus diseases, anatomical structures, and residual anatomical structures were evaluated separately and are summarized in Table 2. CT findings, such as lateralization of the middle turbinate, septal deviation, incomplete uncinectomy, and incomplete anterior-posterior ethmoidectomy, were also present in preoperative physical examination and intraoperative findings.

The following results about relationship between sinus disease or residual anatomical structure with residual cells or number of previous surgeries were identified and summarized as follows:
Table 1: Comorbidities in Patients Undergoing Revision ESS ( $n=83)$.

\begin{tabular}{lcc}
\hline Comorbidity & $\mathbf{n}$ & $\mathbf{\%}$ \\
\hline Allergy & 3 & 3.6 \\
Allergic Rhinitis & 1 & 1.2 \\
Asthma & 15 & 18.1 \\
Asthma + Allergy & 1 & 1.2 \\
Asthma + Allergy & 7 & 8.4 \\
Atopy & 1 & 1.2 \\
DM & 3 & 3.6 \\
Goitre & 1 & 1.2 \\
Goitre + HT & 1 & 1.2 \\
HT & 3 & 3.6 \\
HT + Asthma & 1 & 1.2 \\
HT + HI & 3 & 3.6 \\
Samter's Triad & 2 & 2.4 \\
Without Comorbidity & 41 & 49.4 \\
\hline
\end{tabular}

Table 2: Preoperative CT Findings in Patients Undergoing Revision ESS ( $n=83)$.

\begin{tabular}{|c|c|c|}
\hline Finding & $\mathbf{n}$ & $\%$ \\
\hline \multirow[t]{2}{*}{ Right frontal disease } & Agenesis -2 & 2.4 \\
\hline & 64 & 77.1 \\
\hline \multirow[t]{2}{*}{ Left frontal disease } & Agenesis -1 & 1.2 \\
\hline & 70 & 84.3 \\
\hline Right maxillary disease & 72 & 86.7 \\
\hline Left maxillary disease & 74 & 89.2 \\
\hline Left ethmoid disease & 82 & 98.8 \\
\hline Right ethmoid disease & 83 & 100.0 \\
\hline Right sphenoid disease & 49 & 59.0 \\
\hline Left sphenoid disease & 51 & 61.4 \\
\hline \multirow[t]{2}{*}{ Middle turbinate lateralization - right } & Resected -2 & 2.4 \\
\hline & 26 & 31.3 \\
\hline \multirow[t]{2}{*}{ Middle turbinate lateralization - left } & Resected -2 & 2.4 \\
\hline & 29 & 34.9 \\
\hline Incomplete uncinectomy - right & 23 & 27.7 \\
\hline Incomplete uncinectomy - left & 21 & 25.3 \\
\hline Unopened agger nasi - right & 29 & 34.9 \\
\hline Unopened agger nasi - left & 29 & 34.9 \\
\hline Stenosis of maxillary antrostomy - right & 1 & 1.2 \\
\hline Stenosis of maxillary antrostomy - left & 1 & 1.2 \\
\hline \multirow[t]{2}{*}{ Frontal recess cicatrization - right } & Agenesis -1 & 1.2 \\
\hline & 18 & 21.7 \\
\hline \multirow[t]{2}{*}{ Frontal recess cicatrization - left } & Agenesis -1 & 1.2 \\
\hline & 21 & 25.3 \\
\hline Incomplete anterior ethmoidectomy - right & 32 & 38.6 \\
\hline Incomplete anterior ethmoidectomy - left & 32 & 38.6 \\
\hline Incomplete posterior ethmoidectomy - right & 67 & 80.7 \\
\hline Incomplete posterior ethmoidectomy - left & 67 & 80.7 \\
\hline Sphenoid ostium stenosis - right & 4 & 4.8 \\
\hline Sphenoid ostium stenosis - left & 4 & 4.8 \\
\hline Hyperostosis & 11 & 13.3 \\
\hline \multirow[t]{2}{*}{ Septal deviation } & Right -18 & 21.7 \\
\hline & Left - 29 & 34.9 \\
\hline
\end{tabular}


- There was no statistically significant difference in the number of previous surgeries between patients with asthma (\%28.9) and without asthma. Similar results were also found for 42 patients with comorbidity (\%50.6).

- Moreover, we did not encounter a statistically significant association between the number of previous surgeries and hyperostosis (13.3\%), incomplete right anterior ethmoidectomy $(38.6 \%)$, incomplete left anterior ethmoidectomy (38.6\%), incomplete right posterior ethmoidectomy $(80.7 \%)$, incomplete left posterior ethmoidectomy (80.7\%), or residual ager nasi cell (34.9\%).

- There was no statistically significant difference with right frontal and maxillary disease in patients with right lateralized middle turbinate (\%31.3).

- There was no statistically significant difference with left frontal, ethmoid and maxillary disease in patients with left lateralized middle turbinate (\%34.9).

- There was no association between right residual agger nasi (\%34.9) and right frontal disease, and no association was found on the left side either.

- No statistically significant association was found between left frontal disease and left ethmoid disease; neither did we encounter an association on the right side.

- Statistically significant association was noted between right incomplete uncinectomy and right frontal disease ( $p=0.037)$, and a significant association was also present between left incomplete uncinectomy and left frontal disease $(p=0.039)$.

- No statistically significant associations were observed between septal deviation and both right lateralized middle turbinate and left lateralized middle turbinate.

- Finally, we encountered a statistically significant association between septal deviation and both left $(p=0.002)$ and right (0.002) incomplete posterior ethmoidectomy. This association was not observed for right and left incomplete anterior ethmoidectomy $(p=0.062)$.

\section{DISCUSSION}

Various studies investigating the causes of failure in ESS have been conducted before. In his study in 1992, Kennedy reported that the risk of surgical failure after ESS increased in patients with bilateral sinus disease and diffuse nasal polyposis (8). In a study published in the same year, Lazar et al. stated that the most common intraoperative findings in revision ESS cases were adhesion formation between the middle concha and lateral nasal wall, seen in $43 \%$ of patients, and nasal polyposis recurrence, seen in $22 \%$ of patients (9). Studies by Ramadan (2) and Musy and Kountakis (3) reported that the most common pathological anatomic finding in revision ESS cases was lateralized concha. Khalil et al. (10), on the other hand, evaluated computerized tomography (CT) data of 63 cases scheduled for revision ESS and reported that the most common finding was uncinate process with an incidence rate of $57.1 \%$. They also reported residual anterior-posterior ethmoid cell on the side that requires revision in more than $90 \%$ of cases. In the study conducted by Socher et al. (11) in 2018, CT images of 28 revision ESS patients were evaluated and the most common pathological findings were reported as mucosal thickening in the maxillary sinus (89.28\%) and septal deviation (75\%).

Our study revealed that septal deviation caused inadequate posterior ethmoidectomy in cases requiring revision endoscopic sinus surgery and that incomplete uncinectomy increased the risk of frontal sinus disease. In addition, the relationship between lateralized middle turbinate, which is a common finding in many revision ESS cases, and the frontal, maxillary or ethmoid disease was examined, and no significant correlation was found between these conditions.

The incidence of septal deviation in revision ESS cases is highly variable, ranging from $15.9 \%$ to $75 \%(10,11)$. Septal deviation was detected in 47 (56.6\%) of 83 patients who required revision ESS in our study. While there was no significant difference between septal deviation and lateralized middle turbinate or anterior ethmoidal residual cells, it was found that the risk of encountering right posterior ethmoidal residual cell in cases with right septal deviation and left posterior ethmoidal residual cell in cases with left septal deviation was statistically significant. The deviated septum may unintentionally orient the endoscope laterally, causing the surgeon to assume the skull base position to be more lateral and leave unopened posterior ethmoid cell or cells medially.

When performed poorly, uncinectomy may lead to insufficient visualization of ethmoid and frontal cells and is thought to be one of the important causes of recurrent frontal disease (1, 12). In our study, the rates of incomplete uncinectomy were $27.7 \%$ on the right and $25.3 \%$ on the left. In literature, this rate was found between $7.14 \%$ and $60.3 \%(10,11)$. Reports of such variable rates could be explained by the phenomenon that the uncinate process cannot always be distinguished in CT sections or by the differences in the extent of uncinate resection depending on the differences in surgical techniques (13). In our study, incomplete uncinectomy was found to be associated with frontal disease on both sides; however, no significant correlation was found between maxillary or ethmoid sinus disease. Based on these findings, it can be said that inadequate uncinectomy in interventions on the frontal sinus is associated with persistent or resistant disease in the frontal sinus. This may potentially be caused by further narrowing of the already restricted drainage tract due to ongoing inflammation and subsequent adverse effects on the frontal sinus drainage.

Lateralized middle turbinate is reported to be the most common finding in revision ESS cases in many studies $(2,3,9)$. The possibility of occurrence increases especially in cases where mucosa is not preserved and partial middle turbinate resection is performed (9). In cases requiring revision ESS, the incidence of lateralized middle turbinate probably varies depending on the amount of conservation in different surgical techniques 
$(3,10,11)$. In our study, the relationship between lateralized middle turbinate and the frontal, maxillary, and ethmoid disease was examined separately, but no significant association was found. Based on these findings, it can be interpreted that lateralized middle turbinate alone may not cause ESS failure. On the other hand, each sinus being evaluated individually could lead to the difference from the literature.

Frontal sinus is a very difficult region to operate on due to its variable and complex anatomy, difficult visualization, close proximity to vital structures such as the skull base and orbit, and the need for advanced equipment and experience (12). Scarring in the frontal recess has been shown as the main cause of frontal sinus disease recurrence after ESS (14). Rates of frontal recess obliteration/scar in revision ESS cases are reported to be up to $50 \%$ in literature (3). The rate of rightsided frontal recess cicatrization was $21.7 \%$ in our study and it was $25.3 \%$ on the left side. Although the rate of frontal disease was found to be quite high $(77.1 \%$ on the right and $84.3 \%$ on the left), it was found that frontal recess cicatrization did not increase the risk of frontal disease. The relationship between residual agger nasi/ethmoid cells and frontal disease was also examined and it was found that these did not increase the risk of disease occurrence in the frontal sinus. Considering the findings of our study, it can be suggested that frontal recess cicatrization and residual agger nasi/ethmoid cell presence after ESS alone do not increase the risk of frontal sinus disease.

At least one comorbid disease was found in 42 patients out of 83 enrolled in our study. The most common comorbid disease was asthma, which was present in $18.1 \%$ of patients. Similarly, in the literature, the most common comorbid factor in revision ESS cases is usually asthma, and its rate varies between $9.6 \%$ and $26 \%(2,3)$.

As mentioned above, the reason for high variability in the findings of studies evaluating tomographic data of revision endoscopic sinus surgery cases is the use of more aggressive or more conservative resection techniques. Some authors claim that aggressive resections are necessary to prevent failure in functional ESS, while others believe that preserving normal tissues in the sinus is the key to a good clinical outcome. For instance, there are publications suggesting that the uncinate process, which is usually resected as the first step in functional endoscopic sinus surgery, protects the paranasal sinuses from allergens and should not be resected when possible $(13,15)$. The minimally invasive sinus technique (MIST) described in recent years has been put into practice by many surgeons (16). Preservation of the existing anatomical structure should, of course, be the main goal in every surgery; however, for endoscopic sinus surgery, which is a relatively novel surgical approach, increased preservation rates may cause an increased need for revision surgery.

In our study, unlike other studies in the literature, detailed statistics were compiled on the results of incomplete resection of some structures, such as the relationship between incomplete uncinectomy and maxillary, frontal, and ethmoid disease or the relationship between residual agger nasi and frontal sinus disease. Moreover, a comparison was made between the conditions that are thought to increase ESS failure, such as lateralized middle concha, septum deviation and frontal recess cicatrization, and the pathological conditions they may cause. For instance, the relationship between septum deviation and incomplete ethmoidectomy was evaluated. Thus, the structures and factors that may play a role in ESS failure were examined in detail and important findings were obtained, even though there was no control group in the study.

Unfortunately, there are certain limitations in our study. First, while evaluating the CT images of the patients, Lund-Mackay scoring was not used. Instead, the evaluations were made by scoring yes/no to different structures or disorders in the images. In addition, the patients included in the study were those who had various complaints that continued or just started after ESS. Since the patients who had undergone ESS but did not have residual complaints were not included in the study, there was no control group.

\section{CONCLUSION}

In our study, newly obtained CT scans of patients who required revision endoscopic sinus surgery were evaluated and it was found that septal deviation caused inadequate posterior ethmoidectomy, while incomplete uncinectomy increased the risk of frontal sinus disease. The relationship between lateralized middle turbinate and frontal, maxillary, and ethmoid disease was investigated and no significant association was found. We suggest that septum deviation may cause insufficient visualization, while incomplete uncinectomy may prevent adequate intervention to the frontal sinus and these subsequently play a role in ESS failure.

Financial Support: This research received no specific grant from any funding agency, or from commercial or not-for-profit sectors.

Ethical Standards: Authors assert that all procedures contributing to this work comply with the ethical standards of the relevant national and institutional guidelines on human experimentation and with the Helsinki Declaration of 1975, as revised in 2008.

Peer-Review: Externally peer-reviewed.

Author Contributions: Conception/Design of Study- L.A., C.Ş.; Data Acquisition- B.Ç., A.K.; Data Analysis/Interpretation- Ş.Ç., M.N.K.T.; Drafting Manuscript- A.K., Ş.Ç.; Critical Revision of Manuscript- M.N.K.T.; Final Approval and Accountability- B.Ş., Ş.Ç.

Conflict of Interest: Authors declared no conflict of interest.

Financial Disclosure: Authors declared no financial support.

\section{REFERENCES}

1. Kennedy DW. Functional endoscopic sinus surgery. Technique. Arch Otolaryngol 1985;111(10):643-9.

2. Ramadan $\mathrm{HH}$. Surgical causes of failure in endoscopic sinus surgery. Laryngoscope 1999;109(1):27-9. 
3. Musy PY, Kountakis SE. Anatomic findings in patients undergoing revision endoscopic sinus surgery. Am J Otolaryngol 2004;25(6):418-22.

4. Chandra RK, Palmer JN, Tangsujarittham T, Kennedy DW. Factors associated with failure of frontal sinusotomy in the early follow-up period. Otolaryngol neck Surg Off J Am Acad Otolaryngol Neck Surg 2004;131(4):514-8.

5. Levine CG, Casiano RR. Revision Functional Endoscopic Sinus Surgery. Otolaryngol Clin North Am 2017;50(1):143-64.

6. Stammberger $\mathrm{H}$. Endoscopic endonasal surgery--concepts in treatment of recurring rhinosinusitis. Part II. Surgical technique. Otolaryngol neck Surg Off J Am Acad Otolaryngol Neck Surg 1986;94(2):147-56.

7. Stammberger H. Endoscopic endonasal surgery--concepts in treatment of recurring rhinosinusitis. Part I. Anatomic and pathophysiologic considerations. Otolaryngol neck Surg Off J Am Acad Otolaryngol Neck Surg 1986;94(2):143-7.

8. Kennedy DW. Prognostic factors, outcomes and staging in ethmoid sinus surgery. Laryngoscope 1992;102(12 Pt 2 Suppl 57):1-18.

9. Lazar RH, Younis RT, Long TE, Gross CW. Revision functional endonasal sinus surgery. Ear Nose Throat J 1992;71(3):131-3.

10. Khalil HS, Eweiss AZ, Clifton N. Radiological findings in patients undergoing revision endoscopic sinus surgery: a retrospective case series study. BMC Ear Nose Throat Disord 2011;11:4.
11. Socher JA, Mello J, Baltha BB. Tomographical Findings in Adult Patients Undergoing Endoscopic Sinus Surgery Revision. Int Arch Otorhinolaryngol 2018;22(1):73-80.

12. Tajudeen BA, Adappa ND. Instrumentation in Frontal Sinus Surgery. Otolaryngol Clin North Am 2016;49(4):945-9.

13. Nayak DR, Balakrishnan R, Murty KD. Endoscopic physiologic approach to allergy-associated chronic rhinosinusitis: a preliminary study. Ear Nose Throat J 2001;80(6):390-2, 395-6, 398 passim.

14. Nakayama T, Asaka D, Kuboki A, Okushi T, Kojima H. Impact of residual frontal recess cells on frontal sinusitis after endoscopic sinus surgery. Eur Arch oto-rhino-laryngology Off J Eur Fed OtoRhino-Laryngological Soc Affil with Ger Soc Oto-Rhino-Laryngology - Head Neck Surg 2018;275(7):1795-801.

15. Nayak DR, Balakrishnan R, Murty KD. Functional anatomy of the uncinate process and its role in endoscopic sinus surgery. Indian J Otolaryngol head neck Surg Off Publ Assoc Otolaryngol India 2001;53(1):27-31.

16. Catalano PJ, Strouch M. The minimally invasive sinus technique: theory and practice. Otolaryngol Clin North Am 2004;37(2):401-9, viii. 